\title{
Association between MPHOSPH6 gene polymorphisms and IgA nephropathy risk in a Chinese Han population
}

\author{
Xiaohong Yang ${ }^{1, *}$, Yin Zhang ${ }^{1, *}$, Wenning Li $^{1}$, Yan Su ${ }^{1}$, Dan Niu ${ }^{2}$, Yanni Wang ${ }^{1}$, \\ Haiyang Huang ${ }^{3}$, Hui Han ${ }^{1}$, Daofa Zhang ${ }^{1}$, Maowei Xie ${ }^{1}$, Huiluan Su${ }^{1}$, Wentan Xu${ }^{1}$ \\ and Jiali Wei ${ }^{1}$ \\ ${ }^{1}$ Department of Nephrology, Hainan General Hospital, Haikou Hainan 570311, China \\ ${ }^{2}$ Department of Nephrology, The First Affiliated Hospital of Xi'an Jiaotong University, Xi'an 710061, China \\ ${ }^{3}$ Central Laboratory, Hainan General Hospital, Haikou Hainan 570311, China \\ *These authors have contributed equally to this work \\ Correspondence to: Jiali Wei, email: drjialiwei@163.com \\ Keywords: IgA nephropathy (IgAN), MPHOSPH6, single nucleotide polymorphisms (SNPS), association study \\ Received: May 10, 2017 \\ Accepted: July 03, 2017 \\ Published: August 01, 2017 \\ Copyright: Yang et al. This is an open-access article distributed under the terms of the Creative Commons Attribution License 3.0 \\ (CC BY 3.0), which permits unrestricted use, distribution, and reproduction in any medium, provided the original author and source \\ are credited.
}

\section{ABSTRACT}

Multiple genetic and environmental factors together contribute to the risk of IgA nephropathy (IgAN). MPHOSPH6 play an important role in the recruitment of the exosome to the pre-rRNA. However, to date, little information is found about the association between MPHOSPH6 polymorphisms and the IgAN risk. In this case-control study, we genotyped five single nucleotide polymorphisms (SNPS) in MPHOSPH6 gene in 416 IgAN cases and 495 controls using Sequenom Mass-ARRAY technology and evaluated their association with IgAN using the $\chi 2$ and genetic model analysis. In the allelic model analysis, we determined rs1056654 was associated with a 0.774fold decrease in the risk of IgAN $(95 \% \mathrm{CI}=0.630-0.952 ; p=0.015)$. In the genetic model analysis, we found that the " $\mathrm{C} / \mathrm{C}$ " genotype of rs1056675 was associated with an increased risk of IgAN based on the codominant model (OR $=1.48 ; 95 \% \mathrm{CI}=1.03-$ $2.13 ; p=0.033)$ and recessive model $(O R=1.52 ; 95 \% C I=1.11-2.09 ; p=0.0095)$. The "G/A-A/A" genotype of rs1056654 was associated with a decreased risk of IgAN based on the dominant model $(O R=0.75 ; 95 \% C I=0.58-0.98 ; p=0.032)$ and logadditvie model $(O R=0.78 ; 95 \% C I=0.64-0.96 ; p=0.0188)$. Our data suggested that gene polymorphisms in the MPHOSPH6 may exert influences IgAN susceptibility in a Chinese Han population.

\section{INTRODUCTION}

$\operatorname{Ig}$ A nephropathy $(\operatorname{IgAN})$ is the most common type of primary glomerulonephritis [1], and more than $40 \%$ of IgAN patients will eventually worsen to end stage renal disease [2]. However, the prevalence of IgAN is significantly different among ethnicities. It is most common in individuals of Asian ancestry, with a very high prevalence of 3.7\% [3], but is rare among Africans [4] and is of intermediate prevalence among Europeans (up to $1.3 \%$ ) [5]. It has been widely accepted that multiple genetic and environmental factors together contribute to the risk of IgAN.
In the past decades, several genome-wide association studies (GWAS) have linked a plenty variety of genetic abnormality in the onset and development of IgAN. Among them, complement factor $\mathrm{H}$ related 1 and 3 (CFHR1, CFHR3) [6], tumor necrosis factor (TNFSF13) [7] and a-defensin (DEFA), major histocompatibility complex (MHC) [8] genes polymorphisms were the most common susceptibility genes with the risk of IgAN. Recently, microRNAs such as miR-148b [9], miR-n-9 [10] were associated with the development of IgAN. These results remind us that study the polymorphisms of genes involving in formation of RNA may provide us a better understanding of IgAN. 
Table 1: Characteristics of cases and controls included in this study

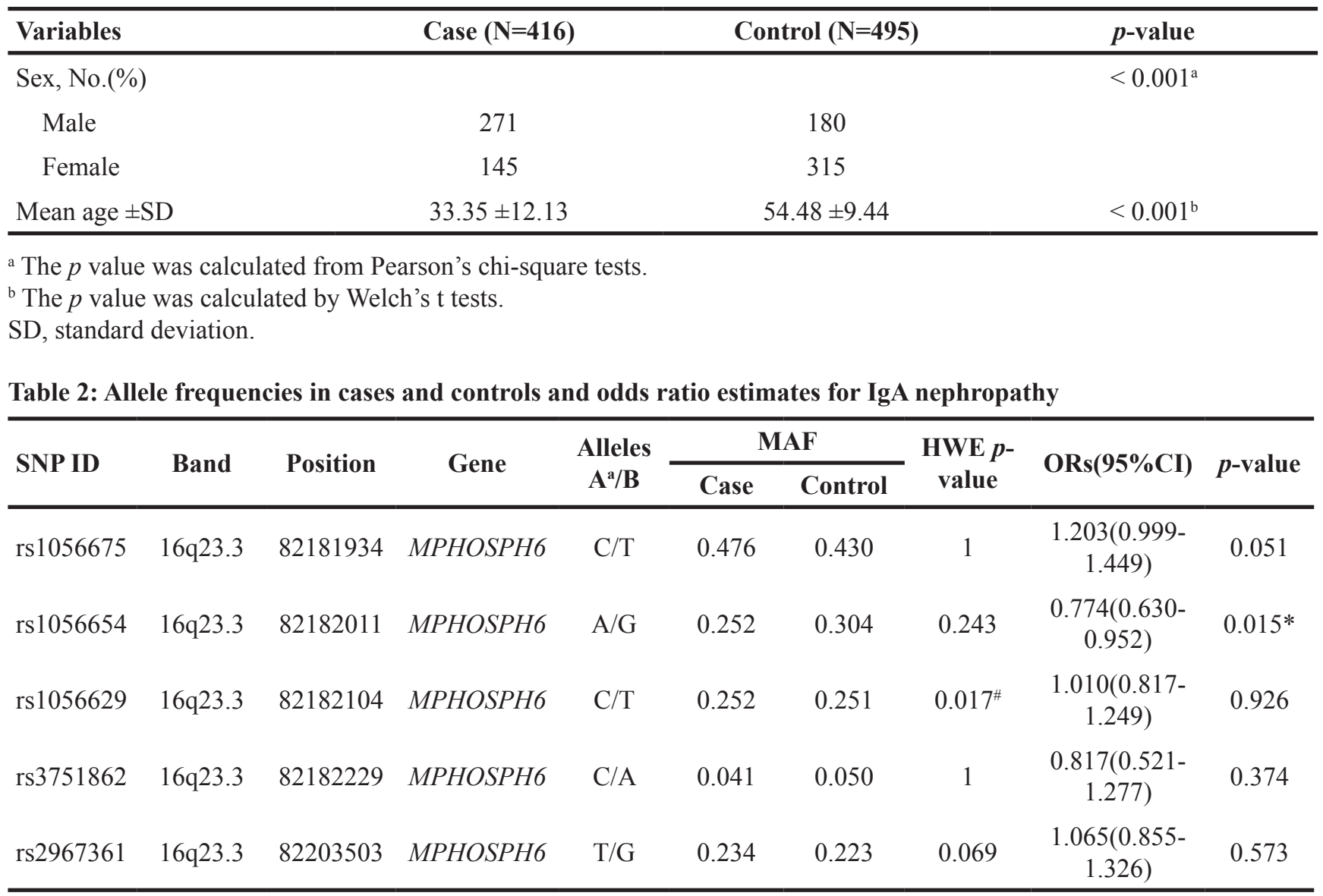

MAF, minor allelic frequency; HWE, Hardy-Weinberg Equilibrium; ORs, odds ratios; CI: confidence interval.

${ }^{a}$ Minor allele; ${ }^{*}$ HWE $p$-value $\leq 0.05$ was excluded; ${ }^{*} p$ value $\leq 0.05$ indicates statistical significance.

It has been demonstrated that MPHOSPH6 play an important role in the recruitment of the exosome to the pre-rRNA [11]. MPHOSPH6 also might be involved in regulating shrimp cell cycle and ovary development [12]. However, to date, little information is found about the association between MPHOSPH6 polymorphisms and the risk of IgAN. In the present study, we selected five single nucleotide polymorphisms (SNPs) of MPHOSPH6 to investigate the association between MPHOSPH6 polymorphisms and the risk of IgAN in a Chinese Han population.

\section{RESULTS}

A total of $416 \operatorname{IgAN}$ cases (271 men and 145 women; mean age, $33.35 \pm 12.13$ years) and 495 controls (180 men and 315 women; mean age, $54.48 \pm 9.44$ years) were included in the study. The basic characteristics of the cases and controls are shown in Table 1.

The MAFs of the analyzed SNPs in the case and control groups are shown in Table 2. All SNPs were in Hardy-Weinberg equilibrium (HWE) in the controls $(p>0.05)$ with the exception of rs 1056629 , which was excluded from subsequent analyses. The MAFs of the SNPs in the control group were similar to those reported for the HapMap Asian population. Using chi-square tests, we determined that rs1056654 was associated with a 0.774 -fold decrease in the risk of $\operatorname{IgAN}(95 \%$ confidence interval $[\mathrm{CI}]=0.630-0.952 ; p=0.015$ ).

The genotype frequencies of the MPHOSPH6 polymorphisms are shown in Table 3. Compared with the TT genotype, the CC frequency of rs 1056675 polymorphism among cases was significantly different from controls (CC versus TT: $\mathrm{OR}=1.48,95 \% \mathrm{CI}=1.03$ 2.13, $p=0.035$ ), which suggested that the rs 1056675 polymorphism had an increased effect on the risk of IgAN. Additionally, we observed that rs 1056654 polymorphism have a protective role of IgAN risk (AA versus GG: OR $=0.61,95 \% \mathrm{CI}=0.38-0.98, p=0.045$ ).

Furthermore, we assumed that the minor allele of each SNP as a risk factor compared with the wild-type allele. Four models (codominant, dominant, recessive, and log-additive) were applied to analyze the associations between the SNPs and risk of IgAN using an unconditional 
Table 3: Genotypes frequencies of the SNPs and their associations with risk of IgA nephropathy

\begin{tabular}{|c|c|c|c|c|c|}
\hline \multirow{2}{*}{ SNP ID } & \multirow{2}{*}{ Genotype } & \multicolumn{2}{|c|}{ Genotype Frequencies } & \multirow{2}{*}{ OR(95\%CI) } & \multirow{2}{*}{$p^{\mathrm{a}}$} \\
\hline & & Case & Control & & \\
\hline \multirow[t]{3}{*}{ rs 1056675} & TT & $126(30.4 \%)$ & $160(32.5 \%)$ & 1.00 & \\
\hline & $\mathrm{CT}$ & $182(44.0 \%)$ & $242(49.0 \%)$ & $0.96(0.71-1.29)$ & 0.765 \\
\hline & $\mathrm{CC}$ & $106(25.6 \%)$ & $91(18.5 \%)$ & $1.48(1.03-2.13)$ & $0.035^{*}$ \\
\hline \multirow[t]{3}{*}{ rs1056654 } & GG & $236(56.7 \%)$ & $245(49.6 \%)$ & 1.00 & \\
\hline & $\mathrm{AG}$ & $150(36.1 \%)$ & $198(40.1 \%)$ & $0.79(0.60-1.04)$ & 0.090 \\
\hline & AA & $30(7.2 \%)$ & $51(10.3 \%)$ & $0.61(0.38-0.98)$ & $0.045^{*}$ \\
\hline \multirow[t]{3}{*}{ rs 3751862} & $\mathrm{AA}$ & $382(91.8 \%)$ & $446(90.3 \%)$ & 1.00 & \\
\hline & $\mathrm{CA}$ & $34(8.2 \%)$ & $47(9.5 \%)$ & $0.85(0.53-1.34)$ & 0.474 \\
\hline & $\mathrm{CC}$ & $0(0 \%)$ & $1(0.2 \%)$ & l & l \\
\hline \multirow[t]{3}{*}{ rs2967361 } & GG & $244(58.7 \%)$ & $306(61.8 \%)$ & 1.00 & \\
\hline & TG & $149(35.8 \%)$ & $157(31.8 \%)$ & $1.19(0.90-1.58)$ & 0.223 \\
\hline & $\mathrm{TT}$ & $23(5.5 \%)$ & $32(6.4 \%)$ & $0.90(0.51-1.58$ & 0.717 \\
\hline
\end{tabular}

SNP: Single nucleotide polymorphism; OR: odds ratio; 95\%CI: 95\% confidence interval.

${ }^{a} p$ values were calculated by unconditional logistic regression analysis with adjustments for age and gender.

$* p \leq 0.05$ indicates statistical significance.

logistic regression analysis with adjustments for age and sex. We found that the " $\mathrm{C} / \mathrm{C}$ " genotype of rs 1056675 was associated with an increased risk of IgAN based on the codominant model $(\mathrm{OR}=1.48 ; 95 \% \mathrm{CI}=1.03-2.13$; $p=0.033)$ and recessive model $(\mathrm{OR}=1.52 ; 95 \% \mathrm{CI}=1.11$ $2.09 ; p=0.0095)$. The "G/A-A/A" genotype of rs 1056654 was associated with a decreased risk of IgAN based on the dominant model $(\mathrm{OR}=0.75 ; 95 \% \mathrm{CI}=0.58-0.98 ; p=0.032)$ and log-additvie model ( $\mathrm{OR}=0.78 ; 95 \% \mathrm{CI}=0.64-0.96$; $p=0.0188$ ) (Table 4).

\section{DISCUSSION}

In this study, we investigated the associations between five selected MPHOSPH6 SNPs and risk of IgAN in the Chinese Han population of Shaanxi Province. We found that rs 1056675 is associated with an increased risk of IgAN, while rs 1056654 has a protective role for IgAN. Our results suggest that the polymorphisms of MPHOSPH6 may play an important role in the risk of IgAN in the Chinese Han population.

MPHOSPH6 is located on chromosome 16q23.3 and encodes the M-phase phosphoprotein 6 (MPP6) that is important for the maturation of 5.8S rRNA. MPP6 is also a RNA-binding protein, which preferentially binds to pyrimidine homopolymers [13]. In fact, little study is found about the association between MPHOSPH6 and disease. $\mathrm{Li}$ et al has attempted to investigate the association between MPHOSPH6 polymorphisms and risk of colorectal cancer, but they have no significant result [14]. In our study, we detected five SNPs of MPHOSPH6, and found that genetic polymorphisms of MPHOSPH6 are associated with IgAN, which may shed a new light on the in-depth study for this gene.

Previous association studies have found many SNPs associated with IgAN risk in some populations; however, little of them were successfully replicated in other populations. Earlier GWAS studies revealed two common variants rs3115573 and rs3130315 in $M H C$ that influence IgAN risk in a British population [15]. Chinese researchers also attempted to investigate SNPs associated with IgAN in the Chinese populations, they identified novel SNPs including rs3803800, rs2738048, rs660895, rs 1794275 and rs2523946 reached a genome-wide significance [8]. It has been revealed that four SNPs including rs9275224, rs2856717, rs9275424 and rs9275596 were significantly associated with IgAN risk in a GWAS replication study in a combined Chinese, European and African-American population and followed by a meta-analysis in 85 world populations [16]. In the present study, we found that rs1056675 and rs1056654 were significantly associated with IgAN risk in a Chinese Han population. As far as we know, we are the first to report the association between MPHOSPH6 polymorphisms and IgAN risk, so the results identified here should be confirmed in further studies.

Our study had several intrinsic limitations. Firstly, the subjects in this study were all Han Chinese who lived in Shaanxi Province. The results identified here 
Table 4: Association between significant SNPs and risk of IgA nephropathy in multiple inheritance models

\begin{tabular}{|c|c|c|c|c|c|c|c|c|}
\hline SNPs & Model & Genotype & Case & Control & OR (95\% CI) & $p$-value & AIC & BIC \\
\hline \multirow{7}{*}{ rs 1056675} & Codominant & $\mathrm{T} / \mathrm{T}$ & $126(30.4 \%)$ & $160(32.5 \%)$ & 1 & \multirow{3}{*}{$0.033^{*}$} & \multirow{3}{*}{1249.7} & \multirow{3}{*}{1264.1} \\
\hline & & $\mathrm{T} / \mathrm{C}$ & $182(44.0 \%)$ & $242(49.1 \%)$ & $0.96(0.71-1.29)$ & & & \\
\hline & & $\mathrm{C} / \mathrm{C}$ & $106(25.6 \%)$ & $91(18.5 \%)$ & $1.48(1.03-2.13)$ & & & \\
\hline & Dominant & $\mathrm{T} / \mathrm{T}$ & $126(30.4 \%)$ & $160(32.5 \%)$ & 1 & \multirow{2}{*}{0.51} & \multirow{2}{*}{1254.1} & \multirow{2}{*}{1263.7} \\
\hline & & $\mathrm{T} / \mathrm{C}-\mathrm{C} / \mathrm{C}$ & $288(69.6 \%)$ & $333(67.5 \%)$ & $1.10(0.83-1.46)$ & & & \\
\hline & \multirow[t]{2}{*}{ Recessive } & $\mathrm{T} / \mathrm{T}-\mathrm{T} / \mathrm{C}$ & $308(74.4 \%)$ & $402(81.5 \%)$ & 1 & \multirow{2}{*}{$0.0095 *$} & \multirow{2}{*}{1247.7} & \multirow{2}{*}{1257.4} \\
\hline & & $\mathrm{C} / \mathrm{C}$ & $106(25.6 \%)$ & $91(18.5 \%)$ & $1.52(1.11-2.09)$ & & & \\
\hline & Log-additive & - & 一 & - & $1.19(0.99-1.43)$ & 0.057 & 1250.9 & 1260.5 \\
\hline \multirow{8}{*}{ rs 1056654} & \multirow[t]{3}{*}{ Codominant } & $\mathrm{G} / \mathrm{G}$ & $236(56.7 \%)$ & $245(49.6 \%)$ & 1 & \multirow{3}{*}{0.06} & \multirow{3}{*}{1255.2} & \multirow{3}{*}{1269.7} \\
\hline & & $\mathrm{G} / \mathrm{A}$ & $150(36.1 \%)$ & $198(40.1 \%)$ & $0.79(0.60-1.04)$ & & & \\
\hline & & $\mathrm{A} / \mathrm{A}$ & $30(7.2 \%)$ & $51(10.3 \%)$ & $0.61(0.38-0.99)$ & & & \\
\hline & Dominant & $\mathrm{G} / \mathrm{G}$ & $236(56.7 \%)$ & $245(49.6 \%)$ & 1 & \multirow{2}{*}{$0.032 *$} & \multirow{2}{*}{1254.2} & \multirow{2}{*}{1263.8} \\
\hline & & $\mathrm{G} / \mathrm{A}-\mathrm{A} / \mathrm{A}$ & $180(43.3 \%)$ & $249(50.4 \%)$ & $0.75(0.58-0.98)$ & & & \\
\hline & \multirow[t]{2}{*}{ Recessive } & G/G-G/A & $386(92.8 \%)$ & $443(89.7 \%)$ & 1 & \multirow{2}{*}{0.098} & \multirow{2}{*}{1256.1} & \multirow{2}{*}{1265.7} \\
\hline & & $\mathrm{A} / \mathrm{A}$ & $30(7.2 \%)$ & $51(10.3 \%)$ & $0.68(0.42-1.08)$ & & & \\
\hline & Log-additive & - & - & - & $0.78(0.64-0.96)$ & $0.0188^{*}$ & 1253.2 & 1262.8 \\
\hline
\end{tabular}

ORs, odds ratios; CI: confidence interval; AIC: Akaike's Information criterion; BIC: Bayesian Information criterion.

$* p$ value $\leq 0.05$ indicates statistical significance.

Table 5: Primers used in this study

\begin{tabular}{lccc}
\hline SNP & 1st_PCR primer & 2nd_PCR primer & UEP_SEQ \\
\hline \multirow{2}{*}{ rs1056675 } & ACGTTGGATGAATACT & ACGTTGGATGGTCAAG & ggtgCGTACATACAAT \\
& TAAGGCTGGAGAGG & CCAATTCGTACATAC & TTGGAATCAA \\
rs1056654 & ACGTTGGATGGTATGT & ACGTTGGATGCAGTCAC & ACCTTGAATTGACT \\
& ACGAATTGGCTTGAC & TGACCTTGAATTG & TACATAAA \\
rs1056629 & ACGTTGGATGTTTTA & ACGTTGGATGGGTCAGT & cGGAAGCAGCC \\
& GCCCCTGATCTAC & GACTGGAGAACTA & CTGTAACAA \\
rs3751862 & ACGTTGGATGTGGTGT & ACGTTGGATGCATCTGTT & TGTTTCTAAAATGATA \\
& CTCTATAGTTATT & TCAAAAACAGC & ATCTCTTTACA \\
& ACGTTGGATGTTACT & ACGTTGGATGAGCTGTA & tCCTGACTGCTT \\
\end{tabular}

UEP_SEQ: Unextended mini-sequencing primer.

need to be confirmed in other ethnicities. Secondly, our sample size was relatively small, so we didn't do further subgroup analyses based on age or gender. Thirdly, IgAN is a heterogeneous disease with many other risk factors. We could not explore the interactions between genetic polymorphisms and environmental factors in IgAN patients due to the limited data. Therefore, the relationship between MPHOSPH6 polymorphisms and IgAN risk must be evaluated in future studies with bigger sample size and different populations.

\section{MATERIALS AND METHODS}

\section{Subjects}

We consecutively recruited 416 IgAN cases and 495 healthy controls from the First Affiliated Hospital of Xi'an 
Jiaotong University from March 2014 to December 2016. All subjects were of northern Han Chinese ancestry and were recruited among local inhabitants of Shanxi province from northern China. All the patients were diagnosed and histologically confirmed to suffer from IgAN according to the renal biopsy, and they had not received any systemic treatment before the time of examination. Besides, we excluded patients with any type of cancer, infection, secondary IgAN (Secondary IgAN is seen most commonly in patients with liver disease or mucosal inflammation, in particular affecting the gastrointestinal tract), other renal diseases or autoimmune diseases. We also excluded control subjects with any chronic disease, conditions involving vital organs (liver, heart, lung and brain), central nervous system-related disease, or aggressive metabolic and endocrinological disease. Peripheral blood was collected from both cases and controls for DNA extraction.

All of the participants provided written informed consent. The Human Research Committee for Approval of Research Involving Human Subjects, the First Affiliated Hospital of Xi' an Jiaotong University, approved the use of human blood samples in this study.

\section{SNP selection and genotyping}

In this study, five SNPs in MPHOSPH6 were selected from DbSNP database (http://www.hapmap.org/ index.html.en) and SNP Consortium database (http:// snp.cshl.org/) for analysis. The lower frequency alleles were coded as the minor allele. All of the SNPs had minor allele frequencies (MAFs) $>5 \%$ in the HapMap Chinese Han Beijing population. Genomic DNA was isolated from whole blood samples using the GoldMagMini Purification Kit (GoldMagCo. Ltd. Xi'an, China), and DNA concentrations were measured using the NanoDrop2000 (Thermo Scientific, Waltham, MA, USA). Sequenom Massarray Assay Design 3.0 softwarewas used to design a multiplexed SNP Mass EXTENDED assay [17-19]. Genotyping was performed on a Sequenom MassARRAY RS1000 platform using the manufacturer's protocol. The PCR primers for each SNP are shown in Table 5. Data management and analysis was performed using the Sequenom Typer 4.0 Software [20, 21].

\section{Statistical analysis}

We used Microsoft Excel (Microsoft Corporation, Redmond, WA, USA) and the SPSS 18.0 statistical package (SPSS, Chicago, IL, USA) to perform statistical analyses. All $p$ values presented in this study were two sided, and $p=0.05$ was considered the cutoff for statistical significance. Differences in the characteristics of the case and control study populations were analyzed using chi-square tests for categorical variables and Welch's t tests for continuous variables. In all analyses, the lower frequency allele was considered to be the 'risk' allele. Control genotype frequencies for each SNP were tested for departure from HWE using Fisher's exact tests. Allele and genotype frequencies in the cases and controls were compared using chi-square tests [22]. Four models (codominant, dominant, recessive, and log-additive) were used to assess the association between each genotype and the risk of IgAN. The effects of the polymorphisms on the risk of IgAN were expressed as odds ratios (ORs) with 95\% confidence interval (CIs), which were calculated using unconditional logistic regression analysis after adjusting for age and gender [23].

\section{CONCLUSIONS}

The present study provided evidence that SNPs in the MPHOSPH6 are associated with IgAN in a Chinese Han population. It is possible that these variants are IgAN risk factors and these data can provide a theoretical foundation for other researchers to further study the association between the MPHOSPH6 gene and IgAN risk in the Chinese Han or other populations.

\section{ACKNOWLEDGMENTS}

This work is supported by National Natural Science Foundation of China (No.81560124), Hainan key research and development projects (ZDYF2017113, ZDYF2017114) and Hainan science and technology planned project of Youth outstanding ability of innovation (201704). We thank the First Affiliated Hospital of Xi'an Jiaotong University to provide samples in this study.

\section{CONFLICTS OF INTEREST}

The authors have no conflicts of interest to report.

\section{REFERENCES}

1. Network WGotIIN, Society tRP, Cattran DC, Coppo R, Cook HT, Feehally J, Roberts IS, Troyanov S, Alpers CE, Amore A, Barratt J. The Oxford classification of IgA nephropathy: rationale, clinicopathological correlations, and classification. Kidney Int. 2009; 76: 534-45.

2. Cai GY, Chen XM. Immunoglobulin A nephropathy in China: progress and challenges. Am J Nephrol. 2009; 30: 268-73.

3. Suzuki K, Honda K, Tanabe K, Toma H, Nihei H, Yamaguchi Y. Incidence of latent mesangial IgA deposition in renal allograft donors in Japan. Kidney Int. 2003; 63: 2286-94

4. Nair R, Walker PD. Is IgA nephropathy the commonest primary glomerulopathy among young adults in the USA? Kidney Int. 2006; 69: 1455-8.

5. Varis J, Rantala I, Pasternack A, Oksa H, Jäntti M, Paunu ES, Pirhonen R.. Immunoglobulin and complement deposition in glomeruli of 756 subjects who had committed 
suicide or met with a violent death. J Clin Pathol. 1993; 46: 607.

6. Gharavi AG, Kiryluk K, Choi M, Li Y, Hou P. Genomewide association study identifies susceptibility loci for IgA nephropathy. Nat Genet. 2011; 43: 321-7.

7. Yang C, Jie W, Yanlong Y, Xuefeng G, Aihua T, Yong G, Zheng L, Youjie Z, Haiying Z, Xue Q. Genome-wide association study identifies TNFSF13 as a susceptibility gene for IgA in a South Chinese population in smokers. Immunogenetics. 2012; 64: 747-53.

8. Yu XQ, Li M, Zhang H, Low HQ, Wei X, Wang JQ, Sun LD, Sim KS, Li Y, Foo JN, Wang W, Li ZJ, Yin XY, et al. A genome-wide association study in Han Chinese identifies multiple susceptibility loci for IgA nephropathy. Nat Genet. 2011; 44: 178-82.

9. Serino G, Sallustio F, Cox SN, Pesce F, Schena FP. Abnormal miR-148b expression promotes aberrant glycosylation of IgA1 in IgA nephropathy. J Am Soc Nephrol. 2012; 23: 814-24.

10. Tan K, Chen J, Li W, Chen Y, Sui W, Zhang Y, Dai Y. Genome-wide analysis of microRNAs expression profiling in patients with primary IgA nephropathy. Genome. 2013; 56: 161-9.

11. Schilders G, Raijmakers R, Raats JM, Pruijn GJ. MPP6 is an exosome-associated RNA-binding protein involved in 5.8S rRNA maturation. Nucleic Acids Res. 2005; 33: 6795.

12. Zhou J, Qiu L, Jiang S, Zhou F, Huang J, Yang L, Su T, Zhang D. Molecular cloning and mRNA expression of M-phase phosphoprotein 6 gene in black tiger shrimp ( Penaeus monodon ). Mol Biol Rep. 2013; 40: 1301.

13. Schilders G, Van DE, Pruijn GJ. C1D and hMtr4p associate with the human exosome subunit PM/Scl-100 and are involved in pre-rRNA processing. Nucleic Acids Res. 2007; 35: 2564.

14. Li G, Li Y, Bai W, Jiang D, Duan X, Jia W, Wang H, Hao Y, Yang Z. MPHOSPH6, ZNF208 and RTEL1 polymorphisms in Chinese Han patients with colorectal cancer. Int J Clin Exp Med. 2016; 9: 12976-12981.

15. Feehally J, Farrall M, Boland A, Gale DP, Gut I, Heath S, Kumar A, Peden JF, Maxwell PH, Morris DL. HLA has strongest association with IgA nephropathy in genome-wide analysis. J Am Soc Nephrol. 2010; 21: 1791-1797.

16. Kiryluk K, Li Y, Sannacherchi S, Rohanizadegan M, Suzuki H, Eitner F, Snyder HJ, Choi M, Hou P, Scolari F. Geographic differences in genetic susceptibility to IgA nephropathy: GWAS replication study and geospatial risk analysis. Plos Genet. 2012; 8: e1002765.

17. Gabriel S, Ziaugra L, Tabbaa D. SNP genotyping using the Sequenom MassARRAY iPLEX platform. Curr Protoc Hum Genet. 2009; Chapter 2: Unit 2.12.

18. Kochl S, Niederstatter H, Parson W. DNA extraction and quantitation of forensic samples using the phenolchloroform method and real-time PCR. Methods Mol Biol. 2005; 297: 13-30.

19. Trembizki E, Smith H, Lahra MM, Chen M, Donovan B, Fairley CK, Guy R, Kaldor J, Regan D, Ward J, Nissen MD, Sloots TP, Whiley DM. High-throughput informative single nucleotide polymorphism-based typing of Neisseria gonorrhoeae using the Sequenom MassARRAY iPLEX platform. J Antimicrob Chemother. 2014; 69: 1526-32.

20. Gabriel S, Ziaugra L, Tabbaa D. SNP genotyping using the Sequenom MassARRAY iPLEX platform. Curr Protoc Hum Genet. 2009: 2.12. 1-2. 6.

21. Thomas RK, Baker AC, DeBiasi RM, Winckler W, LaFramboise T, Lin WM, Wang M, Feng W, Zander T, MacConaill LE. High-throughput oncogene mutation profiling in human cancer. Nat Genet. 2007; 39: 347-51.

22. Adamec C. Example of the use of the nonparametric test. Test X2 for comparison of 2 independent examples. Cesk Zdrav. 1964; 12: 613-9.

23. Bland JM, Altman DG. Statistics notes. The odds ratio. BMJ. 2000; 320: 1468. 